J. Dairy Sci. 99:8622-8632

http://dx.doi.org/10.3168/jds.2016-11076

(C) 2016, THE AUTHORS. Published by FASS and Elsevier Inc. on behalf

of the American Dairy Science Association ${ }^{\circledR}$. This is an open access article under

the CC BY-NC-ND license (http://creativecommons.org/licenses/by-nc-nd/3.0/).

\title{
Short communication: Heat-resistant Escherichia coli as potential persistent reservoir of extended-spectrum $\beta$-lactamases and Shiga toxin-encoding phages in dairy
}

\author{
Roger Marti, ${ }^{*}$ Maite Muniesa, $†$ Michael Schmid,‡ Christian H. Ahrens, $\ddagger$ Javorka Naskova, ${ }^{*}$ \\ and Jörg Hummerjohann*1 \\ ${ }^{*}$ Agroscope, Institute for Food Sciences IFS, Schwarzenburgstrasse 161, CH-3003 Bern, Switzerland \\ †Department of Microbiology, University of Barcelona, 08007 Barcelona, Spain \\ ‡Agroscope, Institute for Plant Production Sciences IPS and Swiss Institute of Bioinformatics, Schloss 1, 8820 Wädenswil, Switzerland
}

\begin{abstract}
Here we report the isolation of heat-resistant Escherichia coli from raw milk cheeses. Detection of the heat-resistance markers $c l p K$ and orfI by PCR was followed by phenotypical confirmation of increased heatresistance. These strains were Shiga toxin-negative and, although several were found to be multidrug resistant, no plasmids encoding extended-spectrum $\beta$-lactamases (ESBL) were found in any of the isolates. The aim of this study was to assess the potential of these strains to acquire ESBL plasmids and a modified Shiga toxinencoding phage. Only 4 ESBL-encoding, heat-sensitive E. coli strains were isolated from 1,251 dairy samples (2/455 raw milk and 2/796 raw milk cheese samples). One incompatibility group FII plasmid (CTX-M-14, $79.0 \mathrm{~kb})$ and 3 incompatibility group I1 plasmids (CTX-M-15, 95.2, 96.1, and $97.8 \mathrm{~kb}$ ) were fully sequenced and de novo assembled. All 4 plasmids are readily transferred to heat-resistant $E$. coli isolates in plate matings $\left(9.7 \times 10^{-5}\right.$ to $3.7 \times 10^{-1}$ exconjugants per recipient) and, to a lesser extent, in milk (up to $7.4 \times 10^{-5}$ exconjugants per recipient). Importantly, the plasmids are stably maintained during passaging in liquid media without antimicrobial pressure. The heat-resistant isolate FAM21805 was also shown to be capable of acting as donor of all 4 ESBL plasmids. In addition, 3 of 11 tested ESBL exconjugants of heatresistant strains were lysogenized by the modified Shiga toxin-encoding phage 933W $\Delta$ stx::gfp::cat. The higher fraction of heat-resistant E. coli (93 of 256 isolates) compared with the estimated $2 \%$ previously predicted based on genomic prevalence of heat resistance genes seems to indicate a selection advantage in the raw milk
\end{abstract}

Received February 23, 2016.

Accepted July 13, 2016.

${ }^{1}$ Corresponding author: joerg.hummerjohann@agroscope.admin.ch cheese production environment. The combination of 2 factors may lead to said advantage: increased survival during thermization of raw milk (heating to subpasteurization temperatures) and increased survival rates during cheese ripening. Should these strains acquire ESBL-encoding plasmids, Shiga toxin-encoding phages, or both, these genetic elements would profit from the selection advantage of their host and become more abundant in this particular environment, which in turn could lead to an increased threat to consumers of raw milk products.

Key words: heat resistance, horizontal gene transfer, Escherichia coli, extended spectrum $\beta$-lactamase, dairy

\section{Short Communication}

Bojer et al. (2010) first described a novel plasmidborne clp ATPase in a persistent, unusually heatresistant nosocomial Klebsiella pneumoniae isolate and designated it ClpK. Shortly thereafter, a chromosomal heat-resistance cluster, including a close homolog of $c l p K$ and the heat-resistance marker orfI (named thr for thermotolerance), was described in Cronobacter sakazakii ATCC 29544, and subsequent screening revealed the presence of orfI and its strict correlation with increased decimal reduction values at $58^{\circ} \mathrm{C}\left(\mathrm{D}_{58}\right)$ in other $E n$ terobacteriaceae, including Escherichia coli (Gajdosova et al., 2011). Another study performed comparative genomic analyses of $29 \mathrm{E}$. coli strains and identified a $\sim 14 \mathrm{~kb}$ locus of heat resistance (LHR), with $>99 \%$ sequence identity to the previously described clusters in Klebsiella pneumoniae and Cronobacter sakazakii; the LHR was found to be present in approximately $2 \%$ of published E. coli whole genomes and genome shotgun sequences (Mercer et al., 2015). In dairy products, in vitro characterization of $E$. coli with regard to cheese production-relevant stresses identified several heatresistant strains isolated from raw milk cheeses (Peng 
et al., 2012). Further studies of these strains confirmed increased heat-resistance in milk at subpasteurization temperatures, as employed during thermization, which is relevant because food safety is increased whereas natural enzymes and the flora of raw milk remain intact to provide the characteristic taste and quality of raw milk cheese (Peng et al., 2013b). Heat-resistant strains also showed increased survival rates during ripening of semihard raw milk cheese (Peng et al., 2013a). The aim of the present study was to identify further heatresistant E. coli isolates from the dairy environment and assess their potential to receive and act as a potential persistent reservoir of antimicrobial resistances and virulence genes (encoding Shiga toxins).

In the course of this study, $E$. coli isolates were PCR screened for heat-resistance markers $c l p K$ (Bojer et al., 2010) and orfI (Gajdosova et al., 2011). Double PCR-positive strains were phenotypically confirmed by heat-inactivation experiments in Luria-Bertani Lennox broth $(\mathbf{L B} ; 10 \mathrm{~g} / \mathrm{L}$ of peptone, $5 \mathrm{~g} / \mathrm{L}$ of yeast extract, $5 \mathrm{~g} / \mathrm{L}$ of $\mathrm{NaCl}, \mathrm{pH}$ 7.0). Strains exhibiting a reduction in colony-forming units of less than 1 log within $30 \mathrm{~min}$ at $55^{\circ} \mathrm{C}$ were considered phenotypically heat-resistant. Antimicrobial resistance (AMR) profiles were determined for 18 agents by disc diffusion experiments according to CLSI (2009), with a focus on $\beta$-lactams (Oxoid, Pratteln, Switzerland). The antimicrobials tested were gentamicin, kanamycin, streptomycin, chloramphenicol, tetracycline, nalidixic acid, ciprofloxacin, trimethoprim, sulfamethoxazol/trimethoprim (19/1), ampicillin (AMP), cefoxitin, cephalothin, cefuroxime, cefotaxime (CTX), cefepime, aztreonam, amoxicillin/ clavulanic acid (20/10), and ertapenem. Phylogenetic groups were determined by quadruplex PCR (Clermont et al., 2013) and multilocus sequence typing was performed with the 7-allele scheme previously described (Wirth et al., 2006; University of Warwick, 2016).

Plasmids of strains encoding extended-spectrum $\beta$-lactamases (ESBL) were isolated using the PureYield Plasmid Maxiprep System (Promega, Dübendorf, Switzerland) according to the manufacturer's instructions. Plasmids were sequenced by Illumina MiSeq paired-end library $(2 \times 250$ bp reads, 550 bp insert size; Microsynth, Balgach, Switzerland) and mate-pair library $(2 \times 300 \mathrm{bp}$, median insert size $3 \mathrm{~kb}$, up to $17 \mathrm{~kb}$; Nextera mate pair sample preparation protocol, made in-house). Nextera adapters were removed using NxTrim (O'Connell et al., 2015) and sequences were de novo assembled with SPAdes v3.6.0 (Nurk et al., 2013). After manual circularization, the sequences were confirmed by mapping the raw reads back to the finished sequence and by an additional check with the error-detection tool NxRepair (Murphy et al., 2015). The average sequence coverage of the assembled plas- mids was between 200 and 5,000 fold. Final assemblies were automatically annotated using the RAST server (Overbeek et al., 2014; RAST, 2015), and manually refined using CLC Genomics Workbench (v8.0.3, Qiagen, Hombrechtikon, Switzerland) and BLAST analysis at NCBI (BLAST, 2016). Refined annotations were exported as GenBank files, sequin format tables created using Artemis (v16.0.0; Rutherford et al., 2000), and submitted using the Sequin submission tool (v14.00; Sequin, 2016).

Horizontal gene transfer (HGT) experiments were done in plate matings, as previously described (Toomey et al., 2009) with slight modifications. Equal volumes $(500 \mu \mathrm{L})$ of donor and rifampicin (RIF)-resistant recipient overnight cultures grown in presence of AMP $(100 \mu \mathrm{g} / \mathrm{mL})$ and RIF $(200 \mu \mathrm{g} / \mathrm{mL})$, respectively, were mixed, centrifuged $\left(12,000 \times g, 2 \mathrm{~min}, 25^{\circ} \mathrm{C}\right)$, and had the supernatant completely removed. Cells were resuspended in $50 \mu \mathrm{L}$ of dilution solution $(8 \mathrm{~g} / \mathrm{L}$ of $\mathrm{NaCl}$, $1 \mathrm{~g} / \mathrm{L}$ of peptone) and spotted on predried LB agar plates (without antimicrobials). Donor and recipient alone served as controls. After $24 \mathrm{~h}$ of static incubation at $37^{\circ} \mathrm{C}$, spots were scraped off, cells were resuspended in $3 \mathrm{~mL}$ of dilution solution, and plated in duplicate (separate dilution series) on LB agar with suitable combinations of antimicrobial agents to select for donors $\left(\mathrm{LB}_{\mathrm{AMP}}, 100 \mu \mathrm{g} / \mathrm{mL}\right)$, recipients $\left(\mathrm{LB}_{\mathrm{RIF}}, 200 \mu \mathrm{g} / \mathrm{mL}\right)$, and exconjugants $\left(\mathrm{LB}_{\mathrm{AMP} / \mathrm{RIF}}, 100 / 200 \mu \mathrm{g} / \mathrm{mL}\right)$. Three biological replicates were performed for each donor or recipient combination and all controls. After overnight incubation at $37^{\circ} \mathrm{C}$, colonies were enumerated and exconjugant frequencies per recipient were calculated. Three exconjugant colonies of each biological replicate were PCR confirmed for presence of the heat-resistance marker gene $c l p K$, thus confirming the identity of the recipient, even though frequencies of spontaneous rifampicin resistant mutants of donors were several orders of magnitude lower than exconjugant frequencies, if detected at all. The HGT experiments in milk were done in the same way with the following alterations: overnight cultures (without antimicrobials) were adjusted to a final concentration of $10^{7} \mathrm{cfu} / \mathrm{mL}$ into $25 \mathrm{~mL}$ of homogenized, UHT-treated milk, mixed by inversion, and statically incubated for $24 \mathrm{~h}$ at $37^{\circ} \mathrm{C}$. All antimicrobials were obtained from Sigma-Aldrich (Buchs, Switzerland).

We tested the stability of ESBL plasmids in their wild-type hosts and in the exconjugants derived from $E$. coli strains FAM21805 and K-12 MG1655. The strains were serially passaged without antimicrobial pressure in $\mathrm{LB}$ medium at $37^{\circ} \mathrm{C}$. Cultures were diluted 1 in 1,000 every $24 \mathrm{~h}$ for the first $72 \mathrm{~h}$ followed by a final dilution of 1 in 1,000 and an incubation of $96 \mathrm{~h}$. Samples were plated in duplicate (separate dilution series) on 
$\mathrm{LB}$ and $\mathrm{LB}_{\mathrm{AMP}}$; the experiment was done in 3 biological replicates.

The chloramphenicol-resistant E. coli K-12 MG1655 (933W $\Delta$ stx::gfp::cat; Quirós et al., 2015) served as the donor of the modified phage $933 \mathrm{~W}$ in plate matings. We used the previously generated 11 pFAM22321 exconjugants as recipients for the phage. Putative lysogens (selected with $\mathrm{LB}_{\mathrm{AMP} / \text { chloramphenicol/RIF }}$, 100/25/100 $\mu \mathrm{g} / \mathrm{mL}$ ) were PCR tested for the presence of $c l p K$ (as described above) and the chloramphenicol resistance cassette (cat) of the modified phage (Serra-Moreno et al., 2006). Also, $w r b A$ was confirmed as the phage insertion site by PCR of the left and right junctions between the phage and bacterial genome (Serra-Moreno et al., 2007).

A total of $256 \mathrm{E}$. coli raw milk cheese isolates were screened for heat-resistance markers $\operatorname{clpK}$ and orfI, 93 (36.3\%) of which were $\operatorname{clpK}$ - and orfI-positive (24 and 9 isolates were single positives, respectively). Of the double PCR-positive isolates, 4 were found not to be clearly heat-resistant as judged by heat-inactivation experiments. The PCR for double-positive strains thus predicted heat resistance in our phenotypic test correctly in $95.7 \%(89 / 93)$ of isolates tested. None of the strains screened encode Shiga toxin. Antimicrobial resistance is one of the greatest threats to public health; specifically, ESBL-producing Enterobacteriaceae are considered a major threat (CDC, 2013) and ESBL are the single most important AMR mechanism of gramnegative bacteria (Bush and Jacoby, 2010). We determined the AMR profiles of 44 double PCR-positive, phenotypically confirmed, heat-resistant strains. Nine of the 18 antimicrobial agents chosen were $\beta$-lactams due to the importance of this family of antimicrobials. Five heat-resistant strains $(11.4 \%)$ are multidrug resistant (MDR), defined as resistant to 3 or more classes of antimicrobials, and $9(20.5 \%)$ are resistant to at least 1 antimicrobial agent, with tetracycline resistance being the most frequent ( 8 strains, 18.2\%). A subset of 30 strains (including all MDR strains) is given in Table 1. None of the heat-resistant strains isolated in our study show ESBL phenotypes. However, heat-sensitive, Shiga toxin-negative, ESBL-encoding E. coli were isolated from both raw milk (2 isolates, 455 samples, $431 \mathrm{E}$. coli isolates; CHROMAgar E.coli, Labo-Life, Pully, Switzerland) and raw milk cheeses (2 isolates, 796 samples, 178 E. coli-positive samples, CHROMAgar ECC, LaboLife). These were all MDR strains in addition to the broad resistance to $\beta$-lactams (Table 1 ).

Plasmids encoding ESBL were found, sequenced, and de novo assembled for all 4 ESBL-encoding strains isolated in our study (Table 2). They each encode a narrow-spectrum TEM-1 $\beta$-lactamase and an aminoglycoside $\mathrm{N}\left(3^{\prime}\right)$-acetyltransferase (aac3-III) in addi- tion to a CTX-M family ESBL. Plasmid pFAM22321 belongs to incompatibility group (Inc) FII and encodes CTX-M-14, whereas the other 3 ESBL plasmids are nearly identical IncI1 plasmids, which harbor CTX-M-15; CTX-M-15 and -14 (in descending order) are the 2 most commonly found ESBL in humans. In food animals, CTX-M-14 is the second most common, whereas CTX-M-15 generally plays a minor role and has only been found recently (Endimiani et al., 2012, Geser et al., 2012, Seiffert et al., 2013). Both CTX-M enzymes are commonly associated with incompatibility groups F and I1, among others (Carattoli, 2009). All IncI1 plasmids in this study feature the IncI shufflon region, which includes alternate PilV C-terminal regions (Brouwer et al., 2015). Plasmid pFAM22871_2 and pFAM23292_2 both encode 7 different tip adhesins ( $\operatorname{shf} A, \operatorname{shf} A^{\prime}, \operatorname{shf} B, \operatorname{shf} B^{\prime}, \operatorname{shf} C$, $s h f C^{\prime}$, and $s h f D^{\prime}$ ), albeit in 2 different arrangements, whereas pFAM23293_1 only encodes the adhesion variants $\operatorname{shf} A, A^{\prime}, C$, and $C^{\prime}$. All 3 plasmids have $\operatorname{shf} A^{\prime}$ as the tip of pilV in the consensus arrangement of the assembly. No reference sequence was required to assemble the shufflon regions, thus the arrangements given can be considered the predominant ones for each plasmid under the conditions used for cultivation and extraction. The 3 IncI1 plasmids are highly similar to pJIE174 (EU418931), a CTX-M-15-encoding plasmid of a patient urine E. coli isolate from Westmead Hospital, Sydney, Australia (phylogenetic group D, ST2495; Zong et al., 2015).

We have obtained complete sequences of additional plasmids for 3 of our ESBL isolates (Table 2). FAM22871 harbors an additional IncFIA/FIB/FII plasmid, pFAM22871_1, which encodes a TEM-1 $\beta$-lactamase as well as streptomycin, tetracycline, and sulfonamide resistance genes. For FAM23292, we found an IncFIA/ FIB/FII plasmid, pFAM23292_1, the only AMR gene of which is a TEM-1 $\beta$-lactamase. None of the sequenced plasmids encodes antibacterial biocide or metal resistance genes. This is important to note, as such resistances can contribute to the spread and maintenance of resistance plasmids in absence of antimicrobials (Pal et al., 2014). The sequences of 3 plasmids have been deposited at GenBank: pFAM22321 (CTX-M-14, TEM1, accession no.: KU288634), pFAM22871_1 (TEM-1, MDR, accession no.: KU355873), and pFAM22871_2 (CTX-M-15, TEM-1, accession no.: KU355874).

We used FAM22321 as a donor of its ESBL plasmid with 11 heat-resistant E. coli strains and E. coli K-12 MG1655 as recipients to broadly illustrate that heat-resistant $E$. coli isolated from dairy are amenable to horizontal gene transfer (Figure 1, A). The other 3 ESBL plasmids (pFAM22871_2, pFAM23292_2, and pFAM23293_1) were mated with recipients FAM21805 
and K-12 MG1655 (Figure 1, A). As proof of principle, HGT in milk was assessed with FAM21805 as a recipient and all 4 ESBL strains as donors (Figure 1, B). In a last step, all 4 FAM21805 exconjugants were used as ESBL plasmid donors with FAM23012 as a recipient (Figure 1, C). Exconjugant frequencies in plate matings were high, ranging from $9.7 \times 10^{-5}$ to $3.7 \times 10^{-1}$ exconjugants per recipient. We found statistically significant differences among various combinations of donor and recipient, as indicated in Figure 1 (one-way ANOVA, Holm-Sidak, $\alpha=0.05$ ). Exconjugant frequencies in milk are several orders of magnitude lower than in plate matings with the same recipient (up to $7.4 \times 10^{-5}$ exconjugants per recipient). With FAM22321 as a donor, HGT in milk was not efficient enough for quantitative evaluation, but exconjugants have been obtained and confirmed without prior enrichment nonetheless. For the other 3 donors, FAM22871, FAM23292, and FAM23293, exconjugant frequencies were $6.0 \times 10^{2}, 7.1$ $\times 10^{2}$, and $1.5 \times 10^{3}$ fold lower in milk than in the corresponding plate mating, respectively. Conjugation with strain FAM22321 always resulted in significantly lower exconjugant frequencies compared with at least one other ESBL donor for the same recipient strain. Even when the donor of all 4 ESBL plasmids was the same strain, FAM21805, significantly fewer exconjugants were obtained with pFAM22321 than with pFAM23293_1 (Figure 1, C).

As strains FAM22871 and FAM23292 each harbor a plasmid encoding a narrow-spectrum $\beta$-lactamase (TEM-1) in addition to the ESBL plasmid, we assessed whether one or both are transferred during plate matings. A biological triplicate of HGT experiments with all 4 ESBL strains into recipient strain FAM21805 was carried out by parallel plating with ampicillin $(100 \mu \mathrm{g} / \mathrm{mL})$ and cefuroxime $(100 \mu \mathrm{g} / \mathrm{mL})$ as $\beta$-lactams. The MIC of cefuroxime for TEM-1-producing $E$. coli isolates is well below the employed concentration (Wu et al., 1994), and selection pressure is thus sufficient to distinguish strains harboring CTX-M-14 or -15 from those harboring TEM-1 only. Exconjugant frequencies evaluated on $\mathrm{LB}_{\mathrm{AMP} / \mathrm{RIF}}$ and $\mathrm{LB}_{\text {cefuroxime/RIF }}$ were not significantly different from each other (2-sided $t$-test, $P \geq 0.40$ ). Also, 20 exconjugant colonies per HGT experiment and replicate (60 in total for each donor) were replicated from $\mathrm{LB}_{\mathrm{AMP} / \mathrm{RIF}}$ onto $\mathrm{LB}_{\text {cefuroxime/RIF }}$ and brilliance $\mathrm{ESBL}$ agar (Oxoid). All colonies regrew, confirming that ESBL plasmids had been transferred in each instance tested. Possible transfer of the TEM-1-encoding plasmids was also assessed. For pFAM22871_1, a screening of exconjugants for tetracycline resistance $\left(\mathrm{LB}_{\text {tetracycline, }}\right.$, $15 \mu \mathrm{g} / \mathrm{mL}$ ) was done; only 1 of 60 exconjugants was tetracycline resistant and received pFAM22871_1. The TEM-1-encoding plasmid pFAM23292_1 features no additional antimicrobial resistances we could use for selection. Therefore, specific primers for its TEM-1 region (p61.1_TEM-1_reg_F: 5'-GACAGGAAGCAAAGCTGAAAGG-3' and p61.1_TEM-1_reg_R: 5'-GTGCCGTCCAAGATCATGATCC-3') and a primer pair specific for the TEM-1 region of pFAM23292_2 (p24.2 TEM-1_reg_F: 5'-TTTTCCTCCCACAGGCCTCG-3' and p24.2_TEM-1_reg_R: 5'-CTCCTGCTGTAGTGTAACCTGC-3') were used to screen for presence of each plasmid in exconjugants (annealing temperature: $64^{\circ} \mathrm{C}$ ). Only the ESBL plasmid pFAM23292_2 was found in exconjugants (20 colonies screened); thus, whereas transfer of the narrow-spectrum $\beta$-lactamaseencoding plasmids of our isolates has been observed in the case of pFAM22871_1, the total HGT frequencies in our experiments are almost exclusively due to transfer of ESBL plasmids. The selection using ampicillin for determination of ESBL exconjugants with the other strains as recipients is thus feasible.

The high frequencies of exconjugants observed in plate matings are reason to expect isolation of ESBLencoding, heat-resistant E. coli strains in the future, especially if these plasmids are maintained in absence of antimicrobial pressure. In general, plasmids can be maintained by several mechanisms: high copy number (and stochastic distribution to daughter cells), toxin-antitoxin (TA) systems, or partitioning proteins (MillionWeaver and Camps, 2014). One or more TA systems have been found on all plasmids except pFAM23293_2 and pFAM23293_3 (small plasmids of 5.1 and $3.2 \mathrm{~kb}$ encoding no AMR, Table 2). As reviewed in Wen et al. (2014), if the encoding plasmid is lost then the TA system could act as postsegregation killing mechanism. The 3 highly similar CTX-M-15-encoding plasmids also harbor the partitioning proteins ParA-ParB, which physically distribute the plasmid into both daughter cells and thus maintain it (Bignell and Thomas, 2001; Table 2). The ratio of ampicillin-resistant colony-forming units to total colony-forming units did not decrease during the entire propagation in liquid medium without antimicrobial pressure for any isolate or exconjugant, even though the total colony-forming units decreased statistically significantly on $\mathrm{d} 7$ compared with at least 1 previous time point for all wild-type strains and exconjugants (one-way ANOVA, Holm-Sidak, $\alpha=0.05$, Figure 2). For plasmid stability experiments of exconjugants with FAM22871 and FAM23292 as donors, we confirmed that only the ESBL plasmid had been transferred (by checking tetracycline resistance by plating and the TEM-1 regions by PCR, respectively). The 4 ESBL plasmids were stably maintained in all E. coli strains under the conditions tested. These experimental findings are in agreement with the in silico evidence of mechanisms for plasmid maintenance. 


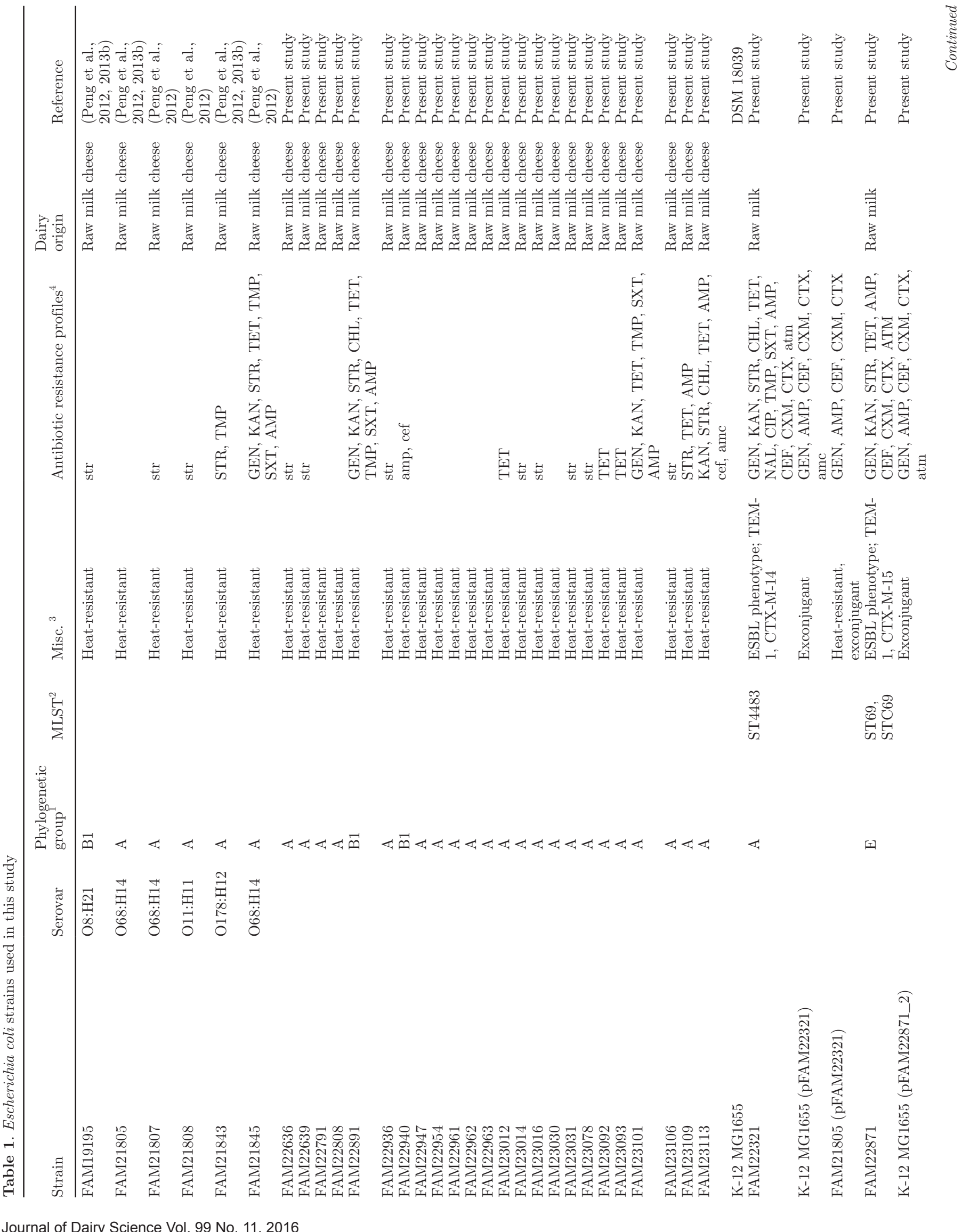




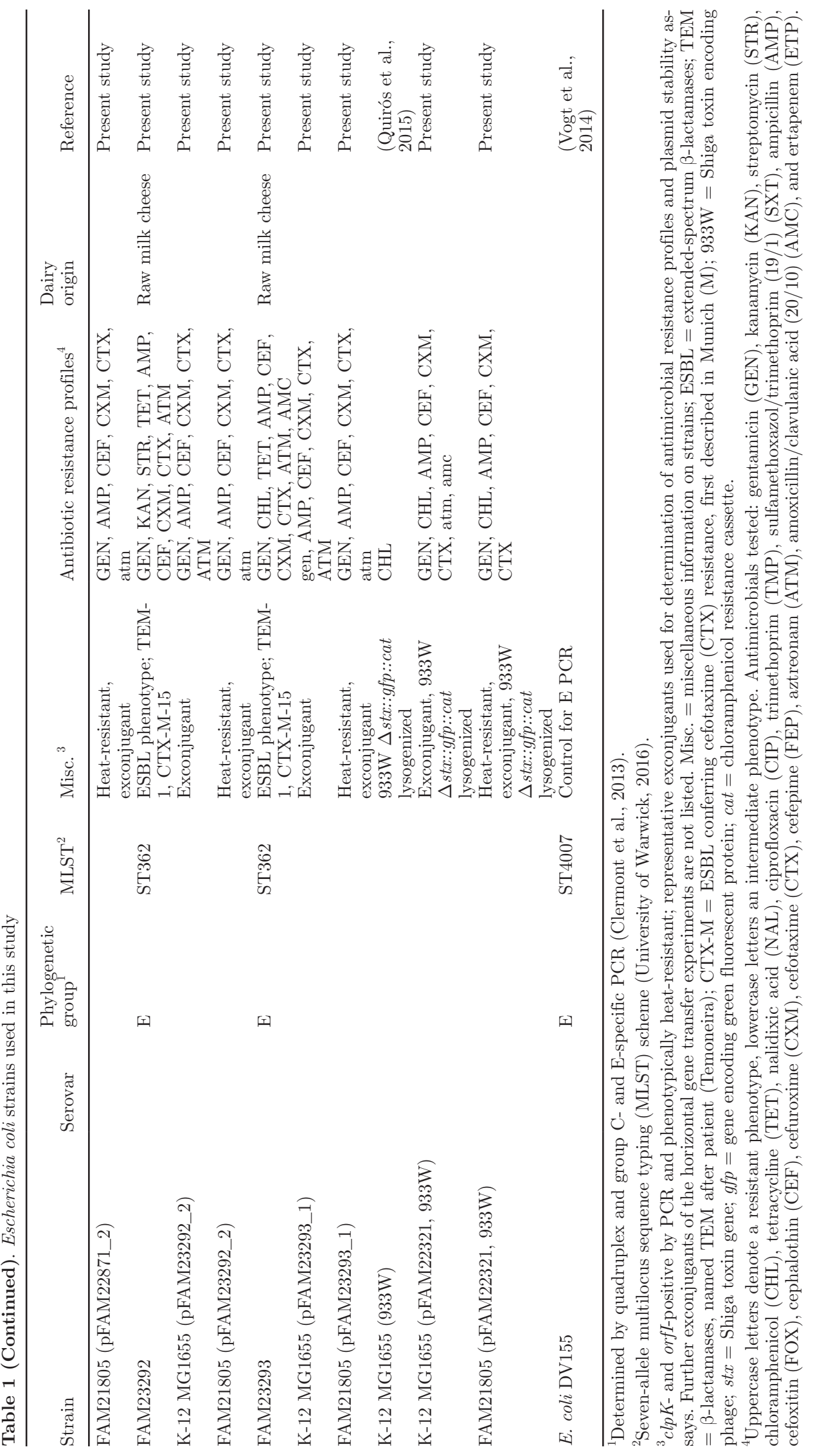


MARTI ET AL.

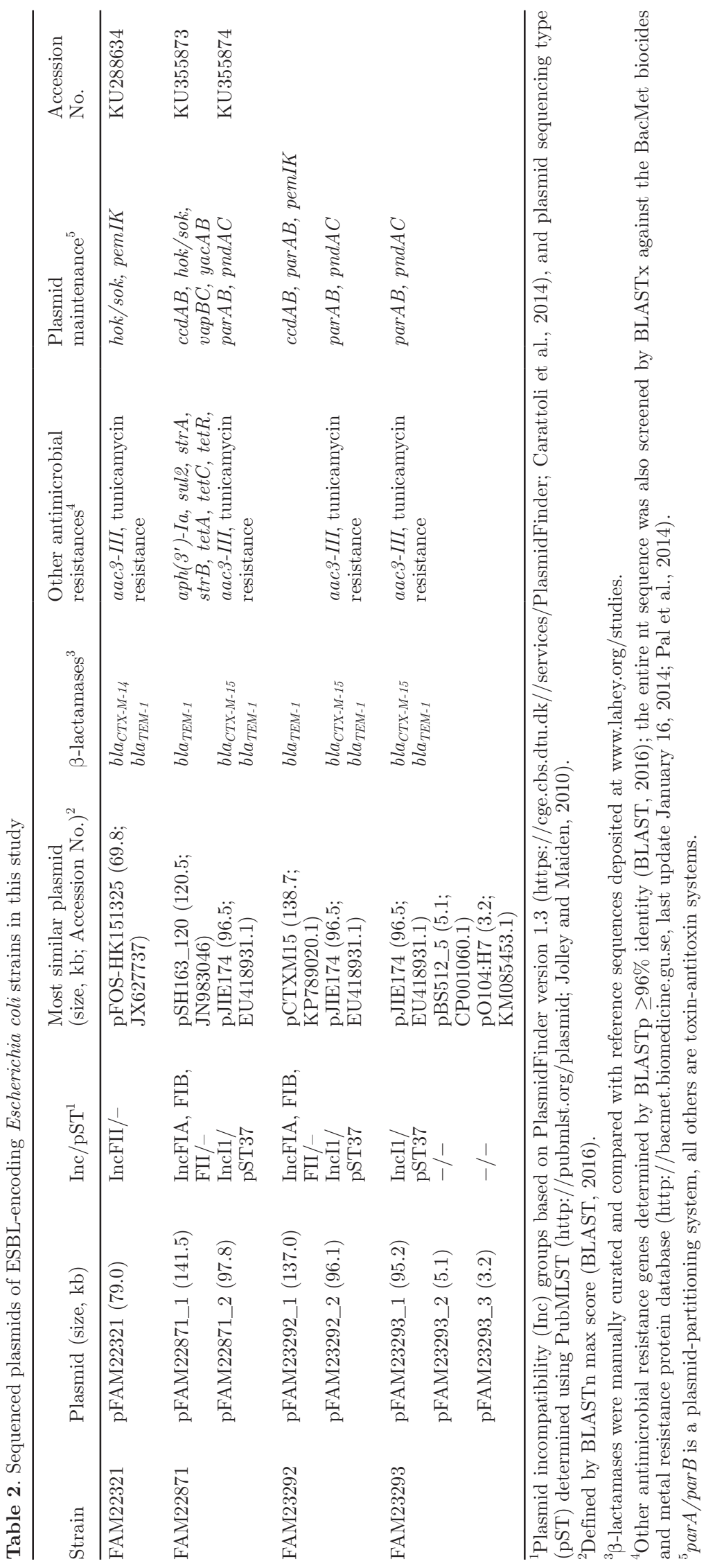


Having demonstrated that heat-resistant E. coli from dairy are capable of efficiently acquiring and maintaining ESBL plasmids, we finally wanted to investigate possible lysogenization by the modified Shiga toxinencoding phage 933W. Lysogenization of exconjugants FAM21805 (pFAM22321), FAM23012 (pFAM22321), and FAM23093 (pFAM22321) was possible in plate matings (PCR-positive for clpK, cat, and left and right junctions), yet frequencies were too close to the detection limits to yield quantitative data (average detection limits of lysogens per recipient: $3.5 \times 10^{-8}, 1.1 \times 10^{-7}$, and $1.5 \times 10^{-7}$ ). The clpK-negative, AMP-, chloramphenicol-, or RIF-resistant colonies were detected as well; these colonies likely were spontaneous RIF-resistant mutants of K-12 MG1655 (933W), which received pFAM22321 from the intended recipient strains.
In this study, we found new heat-resistant E. coli isolates from raw milk cheeses. We found them at a higher rate (93 of 256 isolates) than the approximate $2 \%$ prevalence of the LHR, which includes $c l p K$, in published whole-genome sequences and genome shotgun sequences (Mercer et al., 2015). As the sampling of the current study was done with anonymized samples in collaboration with private diagnostics laboratories, the observed frequency of $36.3 \%$ heat-resistant E. coli possibly does not reflect an exact prevalence value. Still, a true prevalence of more than $2 \%$ is very likely. A possible explanation for this observation is that the milk for production of raw milk cheeses is often treated by thermization of raw milk (heating to subpasteurization temperatures). This increases food safety, but does not denature alkaline phosphatase, and thus distin-

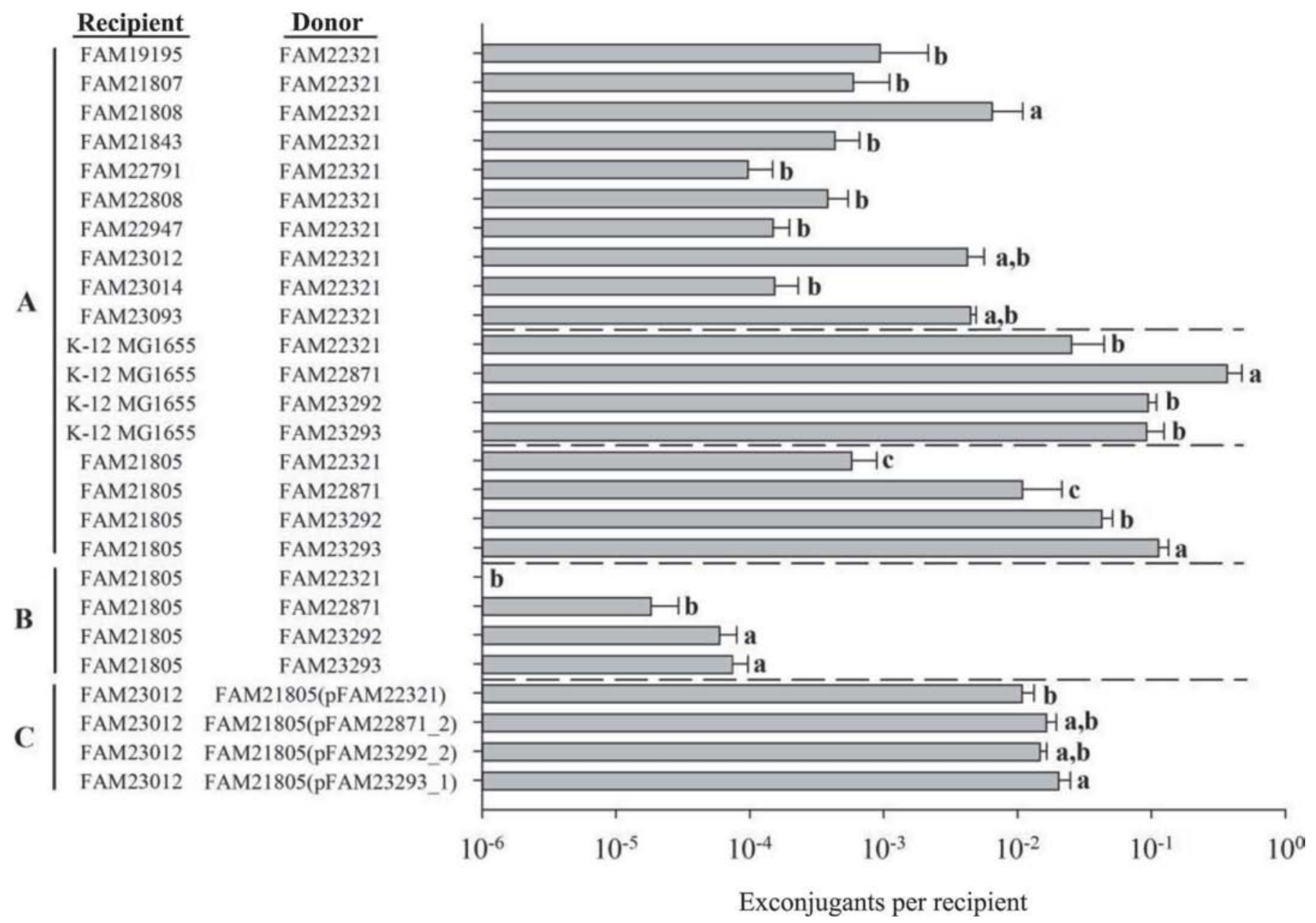

Figure 1. Exconjugant frequencies per recipient. (A) Wild-type strains carrying extended-spectrum $\beta$-lactamases (ESBL)-encoding plasmids FAM22321, FAM22781, FAM23292, and FAM23293 as donors in plate matings. FAM22321 was used as donor for 11 heat-resistant Escherichia coli strains to demonstrate their amenability to horizontal gene transfer and K-12 MG1655 as control. (B) All 4 ESBL-encoding strains as donors in milk. (C) All 4 FAM21805 exconjugants as donors of ESBL-encoding plasmids in plate matings. Bars indicate averages and SD; those not sharing a letter $(\mathrm{a}-\mathrm{c})$ are statistically significantly different from each other. Analyzed by means of one-way ANOVA, Holm-Sidak, $\alpha=0.05$. Groups compared in ANOVA are separated by horizontal dashed lines. 

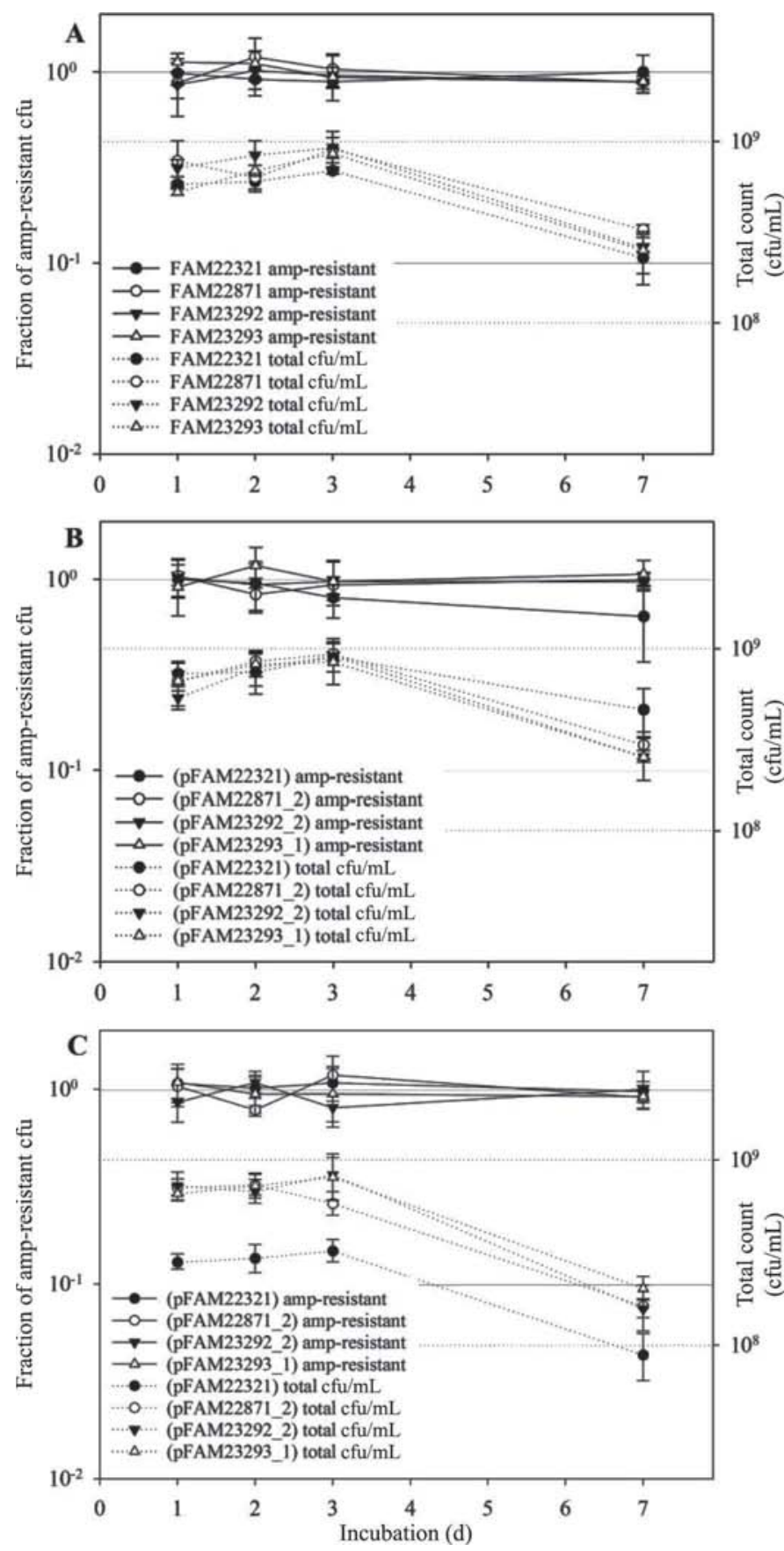

Figure 2. Stability of ESBL plasmids in wild-type strains and exconjugants of FAM21805 and K-12 MG1655 without antimicrobial selection. (A) Stability of plasmids in wild-type extended-spectrum $\beta$-lactamases (ESBL) strains. (B) Stability of FAM21805 exconjugants. (C) Stability of K-12 MG1655 exconjugants. Cultures were diluted 1 to 1,000 each day and incubated at $37^{\circ} \mathrm{C}$ without antimicrobial pressure. The last incubation step was done for $4 \mathrm{~d}$, leading to some decrease in total colony-forming units per milliliter on $\mathrm{d} 7$ (statistically significant to at least one previous time point for all strains tested one-way ANOVA, Holm-Sidak, $\alpha=0.05$ ). No statistically significant differences were found in the ampicillin (amp)-resistant fraction over the time course for any of the strains tested (one-way ANOVA, HolmSidak, $\alpha=0.05)$. Error bars represent SD. guishes these cheeses from those made with pasteurized milk by retaining the unique quality and taste. Several heat-resistant $E$. coli isolates included in our study have previously been shown to exhibit increased decimal reduction values ( $\mathrm{D}$-values) at these subpasteurization temperatures in realistic settings (Peng et al., 2013b). In combination with increased survival rates during cheese ripening (Peng et al., 2013a), this may explain the apparently more frequent isolation of heat-resistant E. coli found in raw milk cheeses. They may be enriched during the entire cheesemaking process compared with their heat-sensitive counterparts. Fortunately, none of the heat-resistant strains was found to be pathogenic or harbor ESBL-encoding plasmids. Only 4 ESBL-encoding E. coli strains were isolated from 1,251 samples in our laboratory so far (455 raw milk and 796 raw milk cheese samples). Geser et al. (2012) also found low prevalence of ESBL-producing E. coli in bulk tank milk (0/100 samples) and mastitis milk (1 of 67 samples encoding CTX-M-14 and TEM-1). None of our ESBL isolates belonged to the invasive $E$. coli sequence type ST131, which is mostly associated with the CTX-M-15 ESBL and contributes significantly to its worldwide spread. In fact, only 1 of the 4 ESBL strains isolated in the current study belongs to a sequence type commonly associated with ESBL-producing E. coli. Strain FAM22871 (CTXM-15) belongs to ST69, which is usually associated with CTX-M-14 (Ewers et al., 2012). Strains FAM23292 and FAM23293 have been isolated from raw milk cheeses of the same dairy 2 mo apart; both belong to phylogenetic group E and are ST362. They are distinguished by the small plasmids pFMA23293_2 and pFAM23293_3, which were not found in FAM23292. All 4 ESBL isolates are capable of conjugating their ESBL plasmids at high rates into heat-resistant $E$. coli, where they are maintained without antimicrobial pressure and remain mobile. This, in conjunction with the likely selection advantage of heat-resistant E. coli during raw milk cheese production, could result in an increased abundance of ESBL plasmids in this system. The strains carrying the ESBL plasmids may then act as donors, as demonstrated with FAM21805 exconjugants. This may happen in the cheese production setting or within the human gastrointestinal tract after consumption, where bacterial densities are high and conjugation is aided by close spatial proximity. This could result in carriage of these plasmids by healthy individuals, with the continuous possibility of pathogens acquiring these plasmids, should an infection be contracted (Machado et al., 2013). As we found for pFAM22321 exconjugants of strains FAM21805, FAM23012, and FAM23093, Shiga toxin phages might also enter the population of heat-resistant E. coli and could profit from a selection advantage. Any kind of reservoir of ESBL or MDR 
plasmids or Shiga toxin-encoding phages, especially if positively selected in a given environment, is worrisome, as it may contribute to the spread of critically important AMR and toxin genes in Enterobacteriaceae.

\section{ACKNOWLEDGMENTS}

Ulrich Zürcher is acknowledged for the management of the Agroscope research program Reduction of Resistant Microorganisms in the Food Chain (REDYMO). This work was financially supported by the Agroscope research programs REDYMO and Microbial Diversity (MicBioDiv). The authors also thank Vincent Perreten (University of Bern, Bern, Switzerland) for kindly providing $E$. coli DV155 strain as control for the phylogenetic group E PCR, Markus Oggenfuss (Agroscope) for help with the mate-pair sequencing, and S. Meier and D. Weik (Agroscope) for technical assistance.

\section{REFERENCES}

Bignell, C., and C. M. Thomas. 2001. The bacterial ParA-ParB partitioning proteins. J. Biotechnol. 91:1-34.

BLAST (Basic Local Alignment Search Tool). 2016. National Center for Biotechnology Information, National Library of Medicine, National Institute of Health, Bethesda, MD. Accessed February 2016. https://blast.ncbi.nlm.nih.gov/Blast.cgi.

Bojer, M. S., C. Struve, H. Ingmer, D. S. Hansen, and K. A. Krogfelt. 2010. Heat resistance mediated by a new plasmid encoded Clp ATPase, ClpK, as a possible novel mechanism for nosocomial persistence of Klebsiella pneumoniae. PLoS ONE 5:e15467 http:// dx.doi.org/10.1371/journal.pone.0015467.

Brouwer, M. S., K. A. Tagg, D. J. Mevius, J. R. Iredell, A. Bossers, H. E. Smith, and S. R. Partridge. 2015. IncI shufflons: Assembly issues in the next-generation sequencing era. Plasmid 80:111-117. http://dx.doi.org/10.1016/j.plasmid.2015.04.009.

Bush, K., and G. A. Jacoby. 2010. Updated functional classification of beta-lactamases. Antimicrob. Agents Chemother. 54:969-976. http://dx.doi.org/10.1128/AAC.01009-09.

Carattoli, A. 2009. Resistance plasmid families in Enterobacteriaceae. Antimicrob. Agents Chemother. 53:2227-2238. http://dx.doi. org/10.1128/AAC.01707-08.

Carattoli, A., E. Zankari, A. Garcia-Fernandez, M. Voldby Larsen, O. Lund, L. Villa, F. Moller Aarestrup, and H. Hasman. 2014. In silico detection and typing of plasmids using PlasmidFinder and plasmid multilocus sequence typing. Antimicrob. Agents Chemother. 58:3895-3903. http://dx.doi.org/10.1128/AAC.02412-14.

CDC. 2013. Antibiotic Resistance Threats in the United States. Centers for Disease Control and Prevention. Accessed September 2015. http://www.cdc.gov/drugresistance/pdf/ar-threats-2013-508.pdf.

Clermont, O., J. K. Christenson, E. Denamur, and D. M. Gordon. 2013. The Clermont Escherichia coli phylo-typing method revisited: improvement of specificity and detection of new phylo-groups. Environ. Microbiol. Rep. 5:58-65. http://dx.doi.org/10.1111/17582229.12019 .

CLSI. 2009. Performance Standards for Antimicrobial Disk Susceptibility Tests; Approved Standard-Tenth Edition. CLSI document M02-A10. Clinical and Laboratory Standards Institute, Wayne, PA.

Endimiani, A., A. Rossano, D. Kunz, G. Overesch, and V. Perreten. 2012. First countrywide survey of third-generation cephalosporinresistant Escherichia coli from broilers, swine, and cattle in Switzerland. Diagn. Microbiol. Infect. Dis. 73:31-38. http://dx.doi. org/10.1016/j.diagmicrobio.2012.01.004.
Ewers, C., A. Bethe, T. Semmler, S. Guenther, and L. H. Wieler. 2012. Extended-spectrum beta-lactamase-producing and AmpC-producing Escherichia coli from livestock and companion animals, and their putative impact on public health: A global perspective. Clin. Microbiol. Infect. 18:646-655. http://dx.doi.org/10.1111/j.14690691.2012.03850.x.

Gajdosova, J., K. Benedikovicova, N. Kamodyova, L. Tothova, E. Kaclikova, S. Stuchlik, J. Turna, and H. Drahovska. 2011. Analysis of the DNA region mediating increased thermotolerance at 58 degrees $\mathrm{C}$ in Cronobacter sp. and other enterobacterial strains. Antonie van Leeuwenhoek 100:279-289. http://dx.doi.org/10.1007/s10482011-9585-y.

Geser, N., R. Stephan, and H. Hachler. 2012. Occurrence and characteristics of extended-spectrum beta-lactamase (ESBL) producing Enterobacteriaceae in food producing animals, minced meat and raw milk. BMC Vet. Res. 8:21. http://dx.doi.org/10.1186/17466148-8-21.

Jolley, K. A., and M. C. Maiden. 2010. BIGSdb: Scalable analysis of bacterial genome variation at the population level. BMC Bioinformatics 11:595. http://dx.doi.org/10.1186/1471-2105-11-595.

Machado, E., T. M. Coque, R. Canton, J. C. Sousa, and L. Peixe. 2013. Commensal Enterobacteriaceae as reservoirs of extended-spectrum beta-lactamases, integrons, and sul genes in Portugal. Front. Microbiol. 4:80. http://dx.doi.org/10.3389/fmicb.2013.00080.

Mercer, R. G., J. Zheng, R. Garcia-Hernandez, L. Ruan, M. G. Ganzle, and L. M. McMullen. 2015. Genetic determinants of heat resistance in Escherichia coli. Front. Microbiol. 6:932. http://dx.doi. org/10.3389/fmicb.2015.00932.

Million-Weaver, S., and M. Camps. 2014. Mechanisms of plasmid segregation: Have multicopy plasmids been overlooked? Plasmid 75:27-36. http://dx.doi.org/10.1016/j.plasmid.2014.07.002.

Murphy, R. R., J. O'Connell, A. J. Cox, and O. Schulz-Trieglaff. 2015. NxRepair: Error correction in de novo sequence assembly using Nextera mate pairs. PeerJ 3:e996. http://dx.doi.org/10.7717/ peerj.996.

Nurk, S., A. Bankevich, D. Antipov, A. A. Gurevich, A. Korobeynikov, A. Lapidus, A. D. Prjibelski, A. Pyshkin, A. Sirotkin, Y. Sirotkin, R. Stepanauskas, S. R. Clingenpeel, T. Woyke, J. S. McLean, R. Lasken, G. Tesler, M. A. Alekseyev, and P. A. Pevzner. 2013. Assembling single-cell genomes and mini-metagenomes from chimeric MDA products. J. Comput. Biol. 20:714-737. http://dx.doi. org $/ 10.1089 / \mathrm{cmb} .2013 .0084$.

O'Connell, J., O. Schulz-Trieglaff, E. Carlson, M. M. Hims, N. A. Gormley, and A. J. Cox. 2015. NxTrim: optimized trimming of Illumina mate pair reads. Bioinformatics 31:2035-2037. http:// dx.doi.org/10.1093/bioinformatics/btv057.

Overbeek, R., R. Olson, G. D. Pusch, G. J. Olsen, J. J. Davis, T. Disz, R. A. Edwards, S. Gerdes, B. Parrello, M. Shukla, V. Vonstein, A. R. Wattam, F. Xia, and R. Stevens. 2014. The SEED and the rapid annotation of microbial genomes using subsystems technology (RAST). Nucleic Acids Res. 42:D206-D214. http://dx.doi. org/10.1093/nar/gkt1226.

Pal, C., J. Bengtsson-Palme, C. Rensing, E. Kristiansson, and D. G. Larsson. 2014. BacMet: Antibacterial biocide and metal resistance genes database. Nucleic Acids Res. 42:D737-D743. http://dx.doi. org/10.1093/nar/gkt1252.

Peng, S., W. Hoffmann, W. Bockelmann, J. Hummerjohann, R. Stephan, and P. Hammer. 2013a. Fate of Shiga toxin-producing and generic Escherichia coli during production and ripening of semihard raw milk cheese. J. Dairy Sci. 96:815-823. http://dx.doi. org/10.3168/jds.2012-5865.

Peng, S., J. Hummerjohann, R. Stephan, and P. Hammer. 2013b. Short communication: Heat resistance of Escherichia coli strains in raw milk at different subpasteurization conditions. J. Dairy Sci. 96:3543-3546. http://dx.doi.org/10.3168/jds.2012-6174.

Peng, S., R. Stephan, J. Hummerjohann, J. Blanco, and C. Zweifel. 2012. In vitro characterization of Shiga toxin-producing and generic Escherichia coli in respect of cheese production-relevant stresses. J. Food Saf. Food Qual. 63:136-141.

Quirós, P., A. Martinez-Castillo, and M. Muniesa. 2015. Improving detection of Shiga toxin-producing Escherichia coli by molecular 
methods by reducing the interference of free Shiga toxin-encoding bacteriophages. Appl. Environ. Microbiol. 81:415-421. http:// dx.doi.org/10.1128/AEM.02941-14.

RAST. 2015. Rapid Annotation using Subsystem Technology. Accessed December 2015. http://rast.nmpdr.org

Rutherford, K., J. Parkhill, J. Crook, T. Horsnell, P. Rice, M. A Rajandream, and B. Barrell. 2000. Artemis: sequence visualization and annotation. Bioinformatics 16:944-945.

Seiffert, S. N., M. Hilty, V. Perreten, and A. Endimiani. 2013. Extended-spectrum cephalosporin-resistant gram-negative organisms in livestock: An emerging problem for human health? Drug Resist. Updat. 16:22-45. http://dx.doi.org/10.1016/j.drup.2012.12.001.

Sequin. 2016. Sequin - A DNA Sequence Submission and Update Tool, National Center for Biotechnology Information, National Library of Medicine, National Institute of Health, Bethesda, MD. Accessed February 2016. http://www.ncbi.nlm.nih.gov/Sequin.

Serra-Moreno, R., S. Acosta, J. P. Hernalsteens, J. Jofre, and M. Muniesa. 2006. Use of the lambda Red recombinase system to produce recombinant prophages carrying antibiotic resistance genes. BMC Mol. Biol. 7:31. http://dx.doi.org/10.1186/1471-2199-7-31.

Serra-Moreno, R., J. Jofre, and M. Muniesa. 2007. Insertion site occupancy by stx2 bacteriophages depends on the locus availability of the host strain chromosome. J. Bacteriol. 189:6645-6654. http:// dx.doi.org/10.1128/JB.00466-07.

Toomey, N., A. Monaghan, S. Fanning, and D. J. Bolton. 2009. Assessment of antimicrobial resistance transfer between lactic acid bacteria and potential foodborne pathogens using in vitro methods and mating in a food matrix. Foodborne Pathog. Dis. 6:925-933 http://dx.doi.org/10.1089/fpd.2009.0278.

University of Warwick. 2016. MLST Databases at UoW. Warwick Medical School, The University of Warwick, Coventry, UK. Accessed February 2016. http://mlst.warwick.ac.uk/mlst/.

Vogt, D., G. Overesch, A. Endimiani, A. Collaud, A. Thomann, and V. Perreten. 2014. Occurrence and genetic characteristics of third-generation cephalosporin-resistant Escherichia coli in Swiss retail meat. Microb. Drug Resist. 20:485-494. http://dx.doi. org/10.1089/mdr.2013.0210.

Wen, Y., E. Behiels, and B. Devreese. 2014. Toxin-antitoxin systems: Their role in persistence, biofilm formation, and pathogenicity. Pathog. Dis. 70:240-249. http://dx.doi.org/10.1111/2049-632X.12145.

Wirth, T., D. Falush, R. Lan, F. Colles, P. Mensa, L. H. Wieler, H. Karch, P. R. Reeves, M. C. Maiden, H. Ochman, and M. Achtman. 2006. Sex and virulence in Escherichia coli: An evolutionary perspective. Mol. Microbiol. 60:1136-1151. http://dx.doi org/10.1111/j.1365-2958.2006.05172.x.

Wu, P. J., K. Shannon, and I. Phillips. 1994. Effect of hyperproduction of TEM-1 beta-lactamase on in vitro susceptibility of Escherichia coli to beta-lactam antibiotics. Antimicrob. Agents Chemother. 38:494-498.

Zong, Z., A. N. Ginn, H. Dobiasova, J. R. Iredell, and S. R. Partridge. 2015. Different IncI1 plasmids from Escherichia coli carry ISEcp1-blaCTX-M-15 associated with different Tn2-derived elements. Plasmid 80:118-126. http://dx.doi.org/10.1016/j. plasmid.2015.04.007. 